\title{
Comparison of head impact measurements via an instrumented mouthguard and an anthropometric testing device
}

\author{
Desney G. Greybe ${ }^{1} \cdot$ Christopher M. Jones $^{1,2} \odot \cdot$ M. Rowan Brown ${ }^{3}$. Elisabeth M. P. Williams ${ }^{1}$
}

Published online: 6 June 2020

(c) The Author(s) 2020

\begin{abstract}
The purpose of this study was to determine and compare the efficacy of head impact measurements via an electronic sensor framework, embedded within a mouthguard, against an anthropometric testing device. Development of the former is in response to the growing issue of head impacts and concussion in rugby union. Testing was conducted in a vehicle safety laboratory using a standard impact protocol utilising the headforms of anthropometric testing devices. The headforms were subjected to controlled front and side impacts. For each impact, the linear acceleration and rotational velocity was measured over a 104-ms interval at a frequency of $1 \mathrm{kHz}$. The magnitude of peak linear acceleration and peak rotational velocity was determined from the measured time-series traces and statistically compared. The peak linear acceleration and rotational velocity had intraclass correlation coefficients of 0.95 and 0.99 , respectively. The root-mean-square error between the measurement systems was $4.3 \mathrm{~g}$ with a standard deviation of $3.5 \mathrm{~g}$ for peak linear acceleration and $0.7 \mathrm{rad} / \mathrm{s}$ with a standard deviation of $0.4 \mathrm{rad} / \mathrm{s}$ for rotational velocity. Bland and Altman analysis indicated a systematic bias of $2.5 \mathrm{~g}$ and $-0.5 \mathrm{rad} / \mathrm{s}$ and limits of agreement $(1.96 \times$ standard deviation $)$ of $\pm 13.1 \mathrm{~g}$ and $\pm 1.25 \mathrm{rad} / \mathrm{s}$ for the instrumented mouthguard. These results provide the basis on which the instrumented mouthguard can be further developed for deployment and application within professional rugby, with a view to accurately and reliably quantify head collision dynamics.
\end{abstract}

\section{Introduction}

Sports-related concussion is one of the most frequent injuries in collision sports, such as, rugby union, American football, ice hockey and boxing, with injury indices reported from $12-33 \%$ for all time-loss injuries [1-3]. Concussive injury and neurocognitive deterioration following repetitive head impacts in these sports has been widely addressed in scientific literature [4-14]. A major theme running through these studies is the need to accurately identify and quantify head impact exposure (HIE), where HIE explicitly refers to the number of head accelerations, above a normal threshold,

Desney G. Greybe and Christopher M. Jones contributed equally to the research.

Christopher M. Jones

Christopher.m.jones@swansea.ac.uk

1 ASTEM, College of Engineering, Swansea University, Swansea, UK

2 Sports and Wellbeing Analytics Limited, Swansea, UK

3 Systems and Process Engineering, College of Engineering, Swansea University, Swansea, UK experienced by an individual participating in contact sports. This number is an important factor in determining any correspondence between sport dependent impact exposure and the immediate and long-term neurocognitive outcome of the individual $[6,11,15]$. As a result, there are many varied studies dedicated to deriving appropriate methods and metrics to quantify and characterize HIE in collision sports [9-11, 15-18].

Measurement methods fall primarily into two categories: (1) video analysis and (2) the analysis of time series data acquired by inertial sensors such as accelerometers and gyroscopes $[6,11,16,19]$. In the former, orthogonal $x y z$ spatial components of well-defined fiducial markers are tracked through a time-series sequence of video frames. This information can then be numerically processed to estimate the dynamics of the markers over the measurement period [19]. In the latter, the orthogonal $x y z$ components of linear acceleration and rotational velocity are measured by the inertial sensors. In both cases, the measurement frame of reference can be translated to a more appropriate inertial frame for HIE analysis, such as the centre of gravity of the head. The data is then analysed to locate the magnitudes of the peak linear acceleration (PLA), peak rotational velocity 
(PRV) and its derivative, the peak rotation acceleration (PRA). These metrics are the primary parameters currently reported and utilised in the literature to quantify $\operatorname{HIE}[6,10$, $11,15,16,20]$.

There are more sophisticated metrics incorporating larger sections of the measured time-series signal, including the Gadd severity index (GSI) [21] and the head injury criterion (HIC) $[22,23]$. For example, $\mathrm{HIC}_{15}$ integrates the acceleration signal over a 15 -ms interval centred around the PLA allowing the shape of the signal to be incorporated within the metric. However, a limiting factor for both GSI and HIC is that they do not account for rotational motion, which has been shown to be a contributory factor in concussive injury [24]. Thus, more recent metrics, such as the rotational injury criterion (rotational equivalent of HIC) and the power rotational head injury criterion explicitly include rotational velocity or acceleration. Furthermore, employing multivariate analysis, Greenwald et al. [25] developed the so-called Head Impact Telemetry Severity Profile (HITSP). The HITSP utilised transformed measures of linear acceleration, rotational acceleration, HIC and GSI, to produce a single score for a given impact. They demonstrated that this measure is more predictive of a concussion event than any one of the single biomechanical measures used to generate it.

Over the past few decades, HIE studies initially focused on helmeted sports, where electronic componentry supporting the operation of the inertial sensors are safely embedded within the helmet. Typically, the inertial sensors are located in the helmet or in an accompanying mouthpiece, which is physically or remotely linked to the electronic infrastructure in the helmet [6, 26, 27]. For example, Greenwald et al. [28] developed the Head Impact Telemetry (HIT) System, which employs a nine-accelerometer array incorporated into a helmet to assess HIE in American football, based on an algorithm for estimating head impacts developed by Crisco et al. [29]. The original HIT system has been adapted and deployed for HIE assessments in a range of other contact sports such as amateur boxing [30], soccer [31] and ice hockey [32].

HIT systems have gone through extensive correlation tests in conjunction with Hybrid III anthropomorphic test device (ATD), including pendulum impact testing [33], helmet-tohelmet collisions [34] and via a linear impactor. Initial work revealed that the mean discrepancy of linear acceleration between the two systems was $4 \%$; however, angular accelerations had a mean error of $17 \%$. Later studies by Beckwith et al. [35] utilising a linear impactor to deliver impacts to an American football helmet found that HIT systems overestimated linear acceleration and underestimated angular acceleration of the Hybrid III ATD headform by $1 \%$ and $6 \%$, respectively. Infield data collected by the HIT systems, and alternatives like the GForceTracker (GForceTracker
Inc., Richmond, Ontario, Canada) [36], have been critical in furthering the understanding of concussion and the development of injury tolerance criteria in many contact sports [37].

More recent research has developed technology to enable quantification of HIE in non-helmeted contact sports such as rugby union, boxing and martial arts [38]. For example, a skin mounted sensor (xPatch; X2 Biosystems, Seattle, Washington USA), which is worn on the mastoid process, comprises a triaxial accelerometer and gyroscope. The skin mounted sensor has been deployed in various contact sports to assess HIE; for example King et al. [16] reported PLA and PRA ranges of $10-153 \mathrm{~g}\left(1 \mathrm{~g}=9.81 \mathrm{~m} / \mathrm{s}^{2}\right)$ and $130-21,890 \mathrm{rad} / \mathrm{s}^{2}$, respectively, with mean values of $17 \mathrm{~g}$ and $2426 \mathrm{rad} / \mathrm{s}^{2}$, respectively, in Australian rules football [16]. A subsequent study by King et al. [39] reported PLA and PRA ranges of $10-123 \mathrm{~g}$ and $89-22,928 \mathrm{rad} / \mathrm{s}^{2}$, respectively, with mean values of $22 \mathrm{~g}$ and $4041 \mathrm{rad} / \mathrm{s}^{2}$ in youth rugby league. Like the HIT systems the skin mounted sensor has been subject to measurement correlation tests; research conducted by Nevins et al. [40] assessed the measurement validity of a skin mounted sensor against a Hybrid III ATD head-neck system. The headform was impacted by pneumatically projected softballs, lacrosse balls and soccer balls at speeds ranging from 10 to $31 \mathrm{~m} / \mathrm{s}$. For low-impact speeds PLA measurements were in agreement; however, the skin mounted sensor underestimated this metric for medium to high-impact speeds. In all scenarios, PRA was underestimated by the skin mounted sensor. Additionally, research by Wu et al. [15] focused on understanding the movement of a skin mounted sensor relative to the underlying bone (soft tissue artefact) during head impacts. Through analysis of highspeed video and skin mounted sensor measurements that captured repeated head-soccer ball impacts (where the ball was projected at $7 \mathrm{~m} / \mathrm{s}$ toward the participants head), they deduced that due to poor skull coupling the skin mounted sensor over-estimated both the PLA and PRV for these moderate collisions.

The work conducted by $\mathrm{Wu}$ et al. [15] also compared the measurement performance of the skin mounted sensor against an instrumented mouthguard; over the past decade these latter devices have increased in electronic complexity. Work by Paris et al. in 2010 [41] instrumented a custom acrylic mouthguard with a single dual-axis accelerometer that could wirelessly transmit linear acceleration data. This design was further developed by Kara et al. [42] to incorporate an array of three accelerometers allowing rotational acceleration to be deduced. More recent mouthguards like those tested and developed by Camarillo et al. [6] and Bartsch et al. [11, 43] utilise triaxial accelerometer and gyroscope inertial sensors each operating at $1 \mathrm{kHz}$, or $4 \mathrm{kHz}$ in the latter case. These advances provided the means to monitor HIE in a variety of sports. For example, the mouthguard tested by Camarillo et al. [6] was deployed 
to monitor 38 New Zealand amateur rugby union players in the 2013 season by King et al. [17]. Similarly, Hernandez et al. [44] employed instrumented mouthguards to measure head impacts of American football players during collegiate games and training. Hernandez et al. [45] also monitored the head impacts of boxers and mixed martial artists, where these data provided a basis on which a finite element human head model [46] was used to reconstruct head collisions.

Like all HIE systems, instrumented mouthguards have been subject to extensive correlation tests. Siegmund et al. [47] assessed the validity of the X2 mouthguard (X2 Biosystems, Seattle, Washington, United States) using a linear impactor to impact a mandibular load-sensing headform [48]. These authors concluded that the X2 mouthguard did not achieve their level of validity for PLA and PRA. Siegmund et al. [47] defined validity as the average intercept and slope not statistically different from zero and one for all impact sites. Camarillo et al. [6] reported normalised RMS errors of $10 \%$ for PLA and PRA when they compared the X2 system against a customised headform mounted on a Hybrid III ATD neck, where collisions were mediated via a linear impactor. Later work on the $\mathrm{X} 2$ system by Wu et al. [15], who assessed the validity whilst measuring football heading impacts with high-speed video, found RMS errors of $16-18 \%$ and $18 \%$ for PLA and PRV, respectively.

The assessment of HIE remains an issue in many contact sports; in professional rugby union, head injuries have resulted in long-term injury, or even retirement in extreme cases. These negative outcomes have intensified media coverage and forced World Rugby (WR), the principal governing body, to revise rules in and around contact [49] in an effort to enhance current player welfare. An instrumented mouthguard (iMG) has the potential to provide unbiased metrology capable of identifying or quantifying HIE sustained by an individual player during a game, a season or even a career. This solution also complies with WR regulation 12 [50], which prohibits clothing or skin based sensors.

In this study, the authors develop a pendulum-based setup to assess the measurement accuracy of inertial sensors (triaxial accelerometer and gyroscope) implanted within an iMG against high specification sensors embedded within Hybrid III and EuroSID-1 ATDs. The iMG was specifically designed and developed for deployment in rugby union, for safety, comfort and power management. The pendulumbased set-up delivered PLA, PRV ranges of 7.0-102.5 g, 6.4-37.0 rad/s, and corresponding impact durations for PLA of $12.1 \pm 5.8-23.5 \pm 6.7 \mathrm{~ms}$, respectively; these ranges are indicative of those previously recorded in rugby union [17]. Differences in PLA and PRV measurements were statistically compared, as was the RMS error of the linear accelerations and rotational velocity time-series traces measured by the two systems.

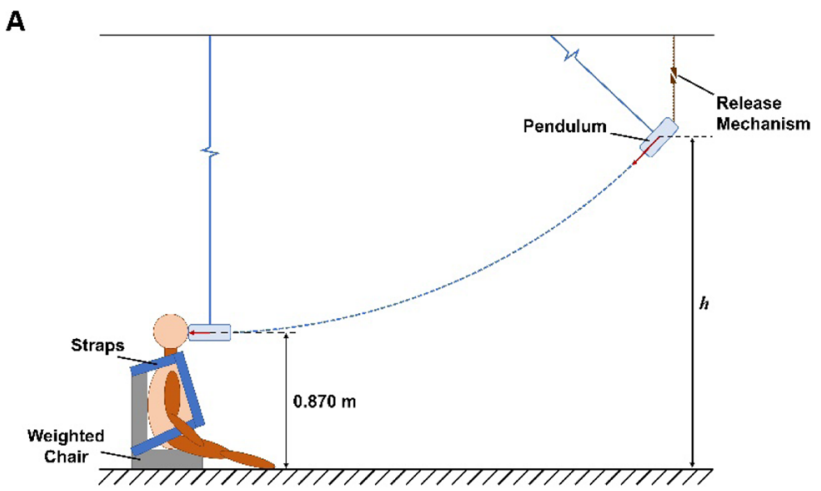

B

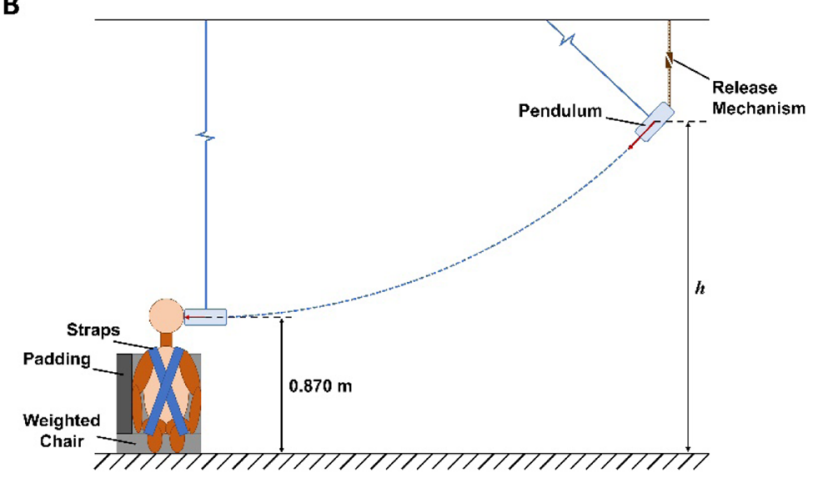

Fig. 1 Schematic of the experimental set-up using the frontal (a) and side (b) impact ATDs. The ATD was strapped onto a weighted chair, with the position of the chair marked on the floor. A pendulum $(8.69$ or $12.89 \mathrm{~kg})$ was raised towards the ceiling and released from predetermined heights, impacting the centre of the ATD headform $0.870 \mathrm{~m}$ above the ground. Preliminary tests were carried out to determine the release heights $(\mathrm{h})$ required for the various impact magnitudes

\section{Materials and methods}

\subsection{Experimental procedure}

Experimental testing was performed in a certified vehicle safety HyGe laboratory (HORIBA MIRA Ltd., Nuneaton, England), utilizing the Hybrid III and EuroSID-1 50th percentile, male ATDs. The Hybrid III has been the industry standard ATD for many years to assess forces imparted during front-on collisions [51]. However, it has been established that the EuroSID-1 is superior to the Hybrid III in assessing lateral forces due to improved design of the thorax, abdomen and shoulder regions [52] and was thus utilised to assess sideimpacts. The laboratory and ATD equipment are considered the vehicle safety testing gold standard, due to the controlled nature of the laboratory and the specifications and calibration of the equipment (see Fig. 1 for experimental set-up).

The ATD torso was secured to a chair with straps, with the chair position marked to ensure consistency of the impact location. The iMG was securely fastened to the horizontal 
A

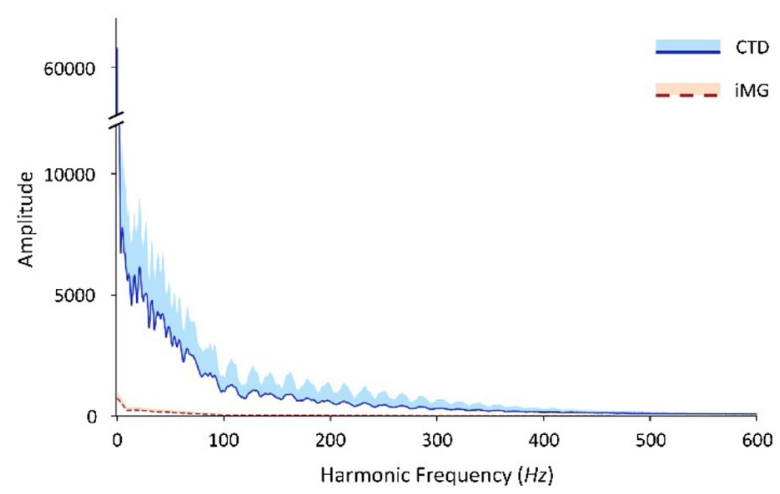

B

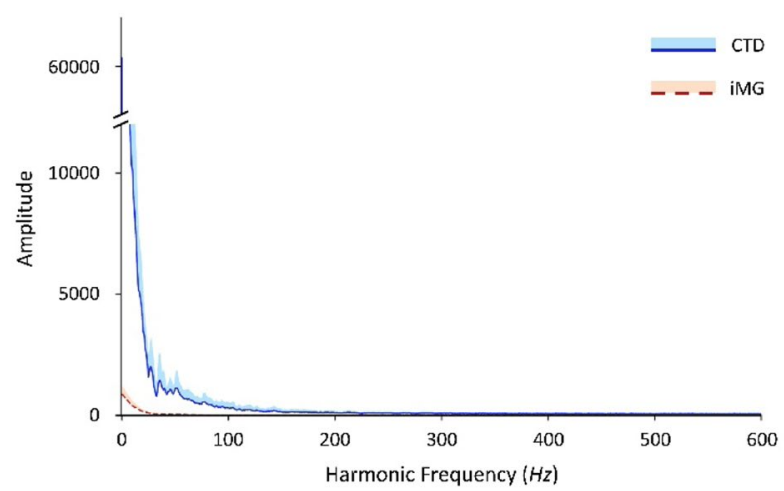

Fig. 2 Fourier frequency transformation of the linear acceleration (a) and angular velocity (b) data. Means are represented by a solid blue (CTD) and broken red (iMG) line. Shaded area represents the mean plus one standard deviation

plate in the centre of the ATD head using high grade duct tape. The tape was then applied over the entire iMG to minimize relative motion with respect to the ATD headform. The coupling was manually checked following each impact and the resultant measurement analysed for high-frequency noise attributed to loose binding (see Fig. 2). Due to the spatial restrictions within the ATD head, the iMG sensor was located $21 \mathrm{~mm}$ anteriorly, $26 \mathrm{~mm}$ laterally and $3 \mathrm{~mm}$ higher than the ATD sensor (see Fig. 3), i.e. there was a displacement vector $\boldsymbol{d}=(21,3,26) \mathrm{mm}$, between the two sensor systems. The relative position of the two sensor systems had no bearing on the final results of the PLA or the PRV, due to the small magnitudes of $\boldsymbol{d}$ and rotational velocities (and associated rotational accelerations) incurred during the impact trials. ${ }^{1}$

\footnotetext{
${ }^{1}$ Given the rigid body transformation for linear acceleration [66], $\boldsymbol{a}_{A}=\boldsymbol{a}_{B}+\boldsymbol{\alpha} \wedge \boldsymbol{d}_{A, B}+\boldsymbol{\omega} \wedge \boldsymbol{\omega} \wedge \boldsymbol{d}_{A, B}$, here the subscripts A and B refer to the iMG and ATD sensors respectively, also $\omega$ and $\alpha$ are the measured rotational velocity and the numerically derived rotational acceleration respectively. Using the above expression, the difference of the
}

A pendulum that comprised of a steel wired arm $(2.5 \mathrm{~m}$ in length) and two flat-faced steel solid cylindrical pendulum heads $(8.69 \mathrm{~kg}$ and $130 \mathrm{~mm}$ diameter; $12.89 \mathrm{~kg}$ and $148 \mathrm{~mm}$ diameter, respectively) were impacted into the ATD headforms to mimic collisions. The pendulums were released from a range of heights (i.e. between 1.387 and $3.139 \mathrm{~m}$ ) above ground level. This set-up was capable of producing PLAs and PRVs of the ATD head between 7.0 and $102.5 \mathrm{~g}$ and 6.4 and $37.0 \mathrm{rad} / \mathrm{s}$, respectively (as recorded by the ATD sensors) at the extremities of the height interval. These peak ranges were consistent with those used in previous validation studies of other instrumented mouthguards $[6,15]$.

\subsection{Sensor specifications}

The ATDs contained a tri-axial accelerometer (Meggitt Sensing Systems Endevco 7264D, range: \pm 2000 g, sensitivity: $0.2 \mathrm{mV} / \mathrm{g}$ ) and a tri-axial gyroscope (Diversified Technical Systems ARS-PRO, range: $\pm 314 \mathrm{rad} / \mathrm{s}$, sensitivity: $\pm 2 \%$ ), located at the centre of gravity of the headform. These sensors recorded at $20 \mathrm{kHz}$, with 16-bit resolution, over a ten second period. The iMG combines a tri-axial accelerometer (H3LIS331DL, STMicroelectronics, Genova, Switzerland) and a tri-axial gyroscope (LSM9DS1, STMicroelectronics, Genova, Switzerland), air tight coated with parylene (Parylene-C, Specialty Coating Systems Ltd, Woking, United Kingdom) and then embedded within the mouthguard by a mouthguard manufacturer (OPRO Ltd, Hemel Hempstead, London, United Kingdom). The former was sampled at $1 \mathrm{kHz}( \pm 200 \mathrm{~g}, 16$-bit resolution) and the latter at $952 \mathrm{~Hz}( \pm 35 \mathrm{rad} / \mathrm{s}, 16$-bit resolution), which were the maximal frequencies of the sensors. The inertial sensors were selected for the iMG based on electronic performance, size, preliminary testing in rugby union and literature assessing HIE in contact sports [16, 53-55]. For each collision, the inertial sensors collect $104 \mathrm{~ms}$ of data from the six channels and store this on the internal central processing unit (CPU memory, CC430F5137IRGZ, Texas Instruments, Dallas, Texas, United States). It is subsequently transmitted to a receiver unit connected to a laptop using a proprietary wireless protocol where it is stored in CSV format for analysis.

\subsection{Data post-processing}

Linear acceleration and rotational velocity time-series data were collected from the iMG and ATD sensors. From these, resultant linear accelerations and rotational velocities were measured. A Fourier frequency transformation was performed for both the iMG and ATD data (see Fig. 2) using

\section{Footnote 1 (continued)}

magnitude of $\boldsymbol{a}_{B}$ at $\boldsymbol{a}_{B}$ was below the sensitivity of the tri-axial accelerometer $( \pm 1 g)$ for all impacts recorded. 
Fig. 3 Schematic of the relative locations of the ATD and iMG sensors in the ATD headform, shown in the frontal (a) and transverse (b) planes. The ATD sensor was fixed to a horizontal plate and located at the centre of mass of the ATD headform. The iMG sensor was located as closely as possible to the ATD sensor. Due to spatial restrictions, the iMG sensor was $26 \mathrm{~mm}$ lateral, $21 \mathrm{~mm}$ anterior and $3 \mathrm{~mm}$ superior to the ATD sensor. The iMG was securely fastened to the horizontal plate using specialist tape, such that no relative movement of the iMG was possible
A

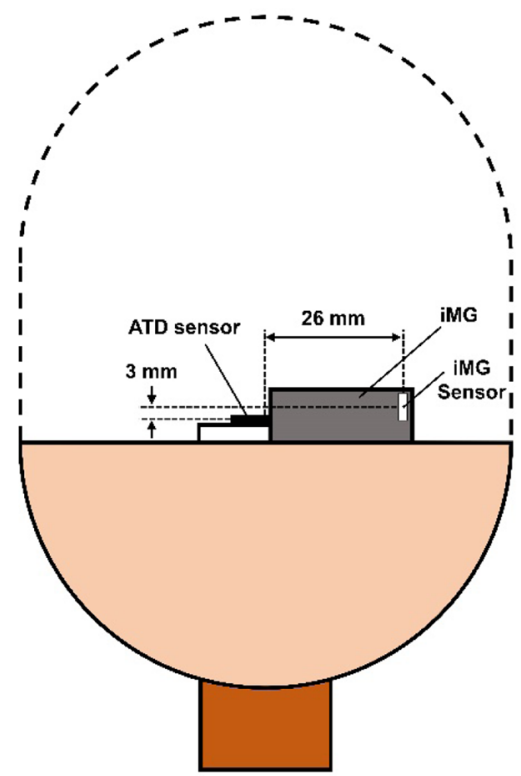

B

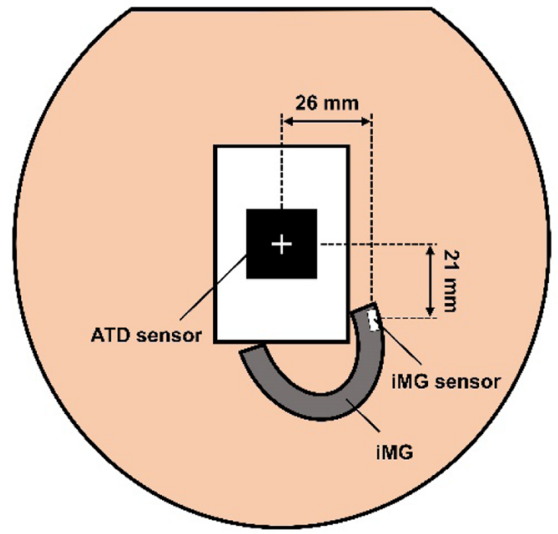

SciPy (v.1.3.0), an open-source Python library for scientific computing [56]. Based on this analysis, there were no obvious high frequency (i.e. $>200 \mathrm{~Hz}$ ) components in the signal measured by the sensors in the iMG, so the data was not filtered. However, a low-pass Butterworth filter was applied to the resultant ATD data to remove high-frequency noise (cut-off frequency of $500 \mathrm{~Hz}$ ), which was attributed to the intrinsic vibration in the experimental system [51].

For each impact, the PLA and PRV were defined as the maximum numerical value of the vector-norm of the respective time-series data. These values were compared between the iMG and ATD sensors using regression analysis. The regression results comparing the ATD data to iMG data were not found to differ between the side and frontal impacts, so all the data was analysed together. Additionally, in alignment with the analysis reported in [6,57], the root mean square (RMS) error was computed between the iMG and ATD time-series data (Eq. 1). The peaks of corresponding impacts were first temporally aligned. They were then time-normalised based on the local minima either side of the impact maximum, which were identified in the ATD data. The RMS errors were also normalised (NRMS) based on the impact magnitude (Eq. 2):

$\mathrm{RMS}=\sqrt{\frac{\sum_{i}^{n}\left(\mathrm{iMG}_{i}-\mathrm{ATD}_{i}\right)^{2}}{n}}$

$\mathrm{NRMS}=\frac{\mathrm{RMS}}{\left(\mathrm{ATD}_{\max }-\mathrm{ATD}_{\min }\right)} \times 100$ where $n$ is the number of measurements, $\mathrm{ATD}_{\max }$ and $\mathrm{ATD}_{\text {min }}$ are the maximum and minimum values recorded by the ATD during the impact.

\subsection{Statistical analysis}

All statistical analysis was performed using SPSS software (Version 25; SPSS Inc., Chicago, IL), with significance set at $p \leq 0.05$. Data was confirmed to be normal and variance was homogenous. An initial reliability study was performed, whereby six trials were recorded at different impact magnitudes. A one-way analysis of variance (ANOVA) was used to determine the reliability between test (trials 1-3) and re-test trials (trials 4-6). Combined and individual coefficient of variation $(\mathrm{CV})$ were determined across the six trials at each impact magnitude for PLA and PRV. The PLA and PRV data were compared for agreement between the iMG and ATD measurements. Intraclass correlation coefficient (ICC) estimates and their $95 \%$ confident intervals (CI) were calculated based on a single-rater, absolute agreement, two-way mixed-effects model. It has been previously reported that a $\mathrm{CV}$ value below $10 \%$ is used to define an acceptable level of reliability $[58,59]$. An ICC above 0.8 was considered a minimum acceptable value for reliability and validity [60]. Scatterplots, with corresponding Pearson correlation coefficients of determination $\left(R^{2}\right)$, were used to evaluate the correlation between measurements. Bland and Altman analysis was conducted to determine the systematic bias and $95 \%$ limits of agreement (LOA) in the data [61]. The measurement error was visualized using Bland and Altman plots, which allowed heteroscedasticity to be assessed [58]. 
Fig. 4 Scatter $(\mathbf{a}, \mathbf{b})$ and Bland and Altman (c, d) plots comparing peak linear acceleration and rotational velocity, measured by the CTD and iMG sensors. a, b Black line is a linear trendline, with the coefficient of determination $\left(R^{2}\right)$ specified. $\mathbf{c}, \mathbf{d}$ Black lines represent the bias and 95\% limits of agreement. Grey areas represent the $95 \%$ confidence intervals for the limits of agreement. Red circled data points denote outliers for linear acceleration and corresponding rotational velocity
A

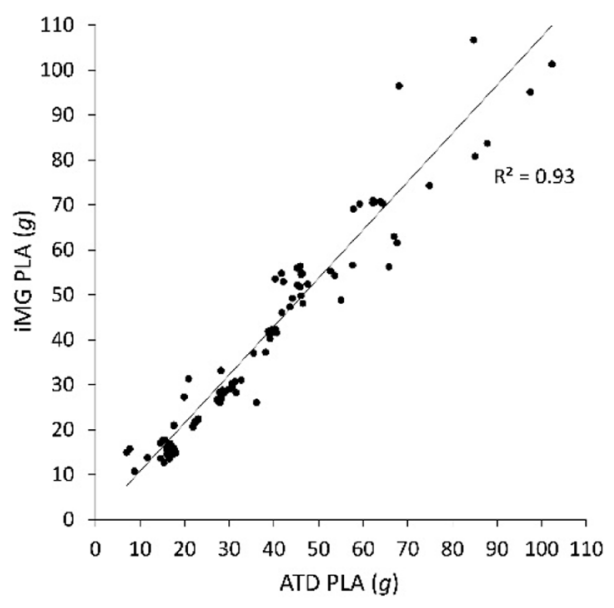

C

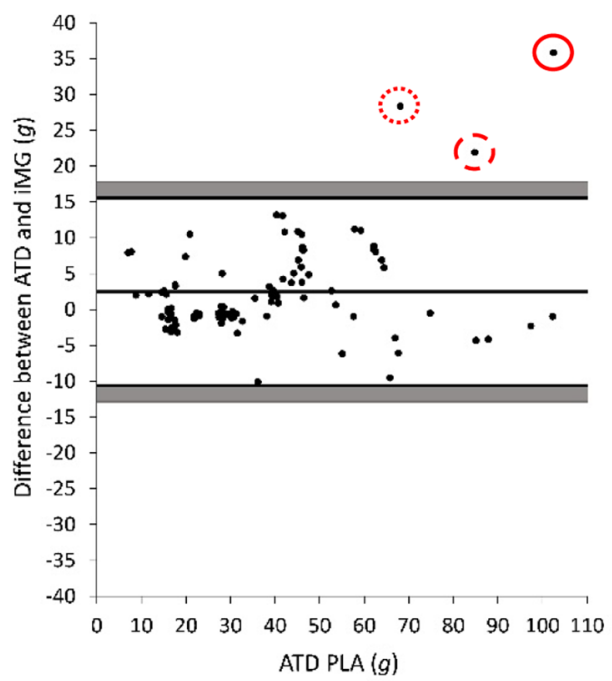

B

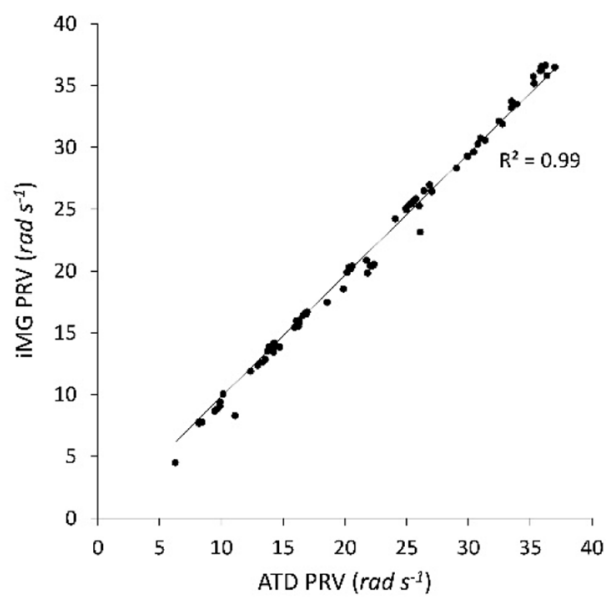

D

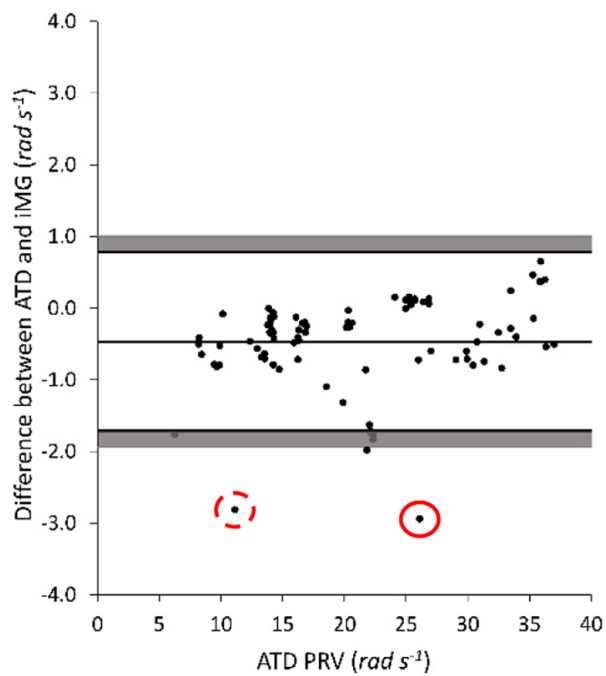

\section{Results}

For all trials, there was no significant difference between test and retest attempts $(p \leq 0.05)$ for both PLA and PRV. Combined CVs were below $<5 \%$, and mean ICC was 0.99 for both PLA and PRV. Figure $4 a-d$ presents the scatter and Bland and Altman plots for the PLA and PRV data, respectively. Figure 4c, d also indicate three outliers within the data set; these correspond to collisions where multiple impacts were observed, by the ATD sensors, during the 104ms measurement interval.

The statistical results are also presented in Table 1, which report the ICC values, $R^{2}$ values, systemic bias and upper and lower limits of agreement, RMS and NRMS. The scatter plots indicate positive correlations between the iMG and ATD measurements, supported by ICCs of 0.95 and 0.99 for PLA and PRV, respectively. The PLA values recorded by the iMG had a systemic bias of $2.5 \mathrm{~g}$ when compared to the ATD sensors with LOA of $\pm 13.1 \mathrm{~g}$. There was greater agreement in the PRV measurements, with a systemic bias of $-0.5 \mathrm{rad} / \mathrm{s}$ and LOA of $\pm 1.25 \mathrm{rad} / \mathrm{s}$.

Representative examples of the time-normalised vectornorms of the measured time-series data are presented in Fig. 5. Specifically, the solid and dashed curves and the dotted and dash-dot curves in Fig. 5a are vector-norms of the linear accelerations as measured via the ATD and iMG sensors, for a 40-g and 30-g impact, respectively. Likewise, the solid and dashed curves and the dotted and dash-dot curves in Fig. 5b are vector-norms of the rotational velocity, as measured via the ATD and iMG sensors, for a 40-g and 30-g impact, respectively. The mean RMS error for linear acceleration, as measured over the impact portion of the time-series data, was $4.3 \mathrm{~g}$ with a standard deviation of $3.5 \mathrm{~g}$ for all impacts. This corresponded to an NRMS error 
Table 1 Table indicating the intraclass correlation coefficients (ICC), their 95\% confidence intervals (CI), coefficients of determination $\left(R^{2}\right)$, systematic bias and the upper and lower $95 \%$ limits of agreement (LOA)

\begin{tabular}{lccccccccc}
\hline & ICC & $(95 \% \mathrm{CI})$ & $R^{2}$ & Systematic bias & Lower LOA & Upper LOA & RMS & $\begin{array}{c}\text { NRMS (\%) } \\
\text { Front impact duration } \\
\text { Lower/upper (ms) }\end{array}$ \\
\hline PLA (g) & 0.95 & $(0.92-0.97)$ & 0.93 & 2.5 & -10.6 & 15.6 & $4.3 \pm 3.5$ & $13.1 \pm 9.9$ & $23.5 \pm 6.7$ \\
PRV ( $\mathrm{rad} / \mathrm{s})$ & 0.99 & $(0.98-0.99)$ & 0.99 & -0.5 & -1.7 & 0.8 & $0.7 \pm 0.4$ & $3.9 \pm 2.8$ & $\ldots$ \\
\hline
\end{tabular}

The root mean square (RMS), normalised root mean square (NRMS) of the vector-norms of the linear acceleration and rotational velocity timeseries traces

*Represent $p<0.05$

A

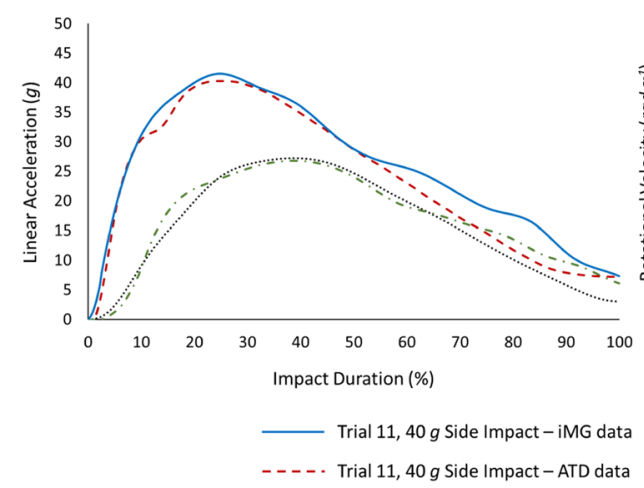

Fig. 5 Examples of the time-normalised impact peaks used to calculate RMS and NRMS errors. The solid and dashed curves and the dotted and dash-dot curves in a are vector-norms of the linear accelerations as measured via the ATD and iMG sensors for a 40-g and

of $13.1 \pm 9.9 \%$. The mean RMS error for rotational velocity was $0.7 \mathrm{rad} / \mathrm{s}$ with a standard deviation of $0.4 \mathrm{rad} / \mathrm{s}$, which corresponded to an NRMS of $3.9 \pm 2.8 \%$.

Table 1 also reports the mean (and standard deviation) of the durations of frontal impacts the lowest and highest release heights (1.387-3.139 m, see Fig. 2) of the pendulum. At the lowest release height, impact durations of $23.5 \pm 6.7 \mathrm{~ms}$ were observed; at the highest release height the impact duration was reduced to $12.1 \pm 5.8 \mathrm{~ms}$. The corresponding impact speeds (and kinetic energies) of the pendulum at these release heights was 2.9 (and $50 \mathrm{~J}$ ) and $6.6 \mathrm{~m} / \mathrm{s}$ (and $287 \mathrm{~J}$ ), respectively.

\section{Discussion}

The results of this study demonstrate systematic agreement for both the linear acceleration and rotational velocity measurements between the iMG and ATD sensor systems. The measured iMG and ATD data were positively correlated, with $R^{2}$ values of 0.96 and 0.98 for PLA and PRV,
B

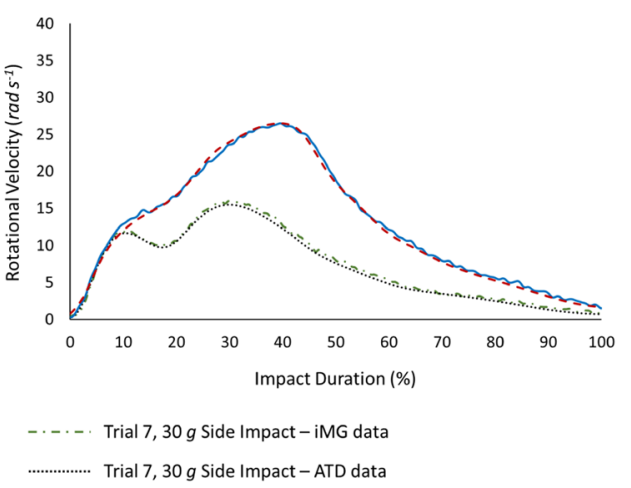

30-g impact, respectively. Likewise, the solid and dashed curves and the dotted and dash-dot curves in $\mathbf{b}$ are vector-norms of the rotational velocity as measured via the ATD and iMG sensors for a $40-\mathrm{g}$ and 30-g impact, respectively

respectively. The corresponding ICC values were 0.95 for PLA and 0.99 for PRV.

The findings of this study are consistent with previous research that has compared the accuracy of other instrumented mouthguards for helmeted collision sports. For example, Camarillo et al. [6] compared a mouthguard using a custom headform and spring-loaded impactor over eight impact velocities $(2.1-8.5 \mathrm{~m} / \mathrm{s})$. They reported $R^{2}$ values of 0.96 and 0.98 for PLA and PRV, respectively. Similarly, Bartsch et al. [11] reported an $R^{2}$ of 0.99 for PLA when comparing their iMG using a head impact dosimeter up to impact velocities of $8.5 \mathrm{~m} / \mathrm{s}$. While these authors did not compare PRV, they reported an $R^{2}$ of 0.98 for PRA. The Bland and Altman results in the present study demonstrate a systematic bias of $2.5 \mathrm{~g}$ for PLA, with $95 \%$ LOA of $\pm 13.1 \mathrm{~g}$. The agreement was greater for PRV, with a systematic bias of $-0.5 \mathrm{rad} / \mathrm{s}$ and $95 \% \mathrm{LOA}$ of $\pm 1.25 \mathrm{rad} / \mathrm{s}$.

There were three outliers within the data set (circled in Fig. 4c), i.e. for impacts where the PLA was greater than $70 \mathrm{~g}$, which correspond to impacts where the release heights were close to or at the maximum release position of the pendulum. At these heights it was established that the swing of the 
pendulum was not always true due to rotations imposed by the release mechanism. This resulted in the pendulum hitting the ATD with a glancing blow instead of one well-defined impact in three cases. This produced multiple impact events in both measurement systems as the pendulum slid past the headform. In these three cases, attempts were made to manually align corresponding maximum $g$ impacts; this methodology was not ideal as the number of impact peaks recorded in the ATD system increased due to its high sampling rate. Consequently, it was not always possible to identify exactly corresponding impact peaks and, in these cases, the nearest temporal event was used. This shortcoming led to an increase of the CV between the two measurement systems, increasing from 1 to $3 \%$ (impacts $<90 \mathrm{~g}$ ) to $10 \%$ (impacts $>90 \mathrm{~g}$ ). Future studies could use sled-based or impactor apparatus to provide greater control over the collision dynamics and mitigate against multiple events being recorded [62, 63].

Previous studies of instrumented mouthguards did not present a Bland and Altman analysis as part of their assessment $[6,11]$. Bland and Altman analyses are important, as they describe the agreement between the measures, not just their correlation. Bartsch et al. [11] did report a mean imprecision error of $3.6 \mathrm{~g}$, which is comparable with the systematic bias reported in this study $(2.5 \mathrm{~g})$. It is also important to evaluate the accuracy of the overall shape of the time-series data [6] as future analysis will utilise more sophisticated metrics to assess the measured time-series. As stated in the introduction, they integrate the measured signal over the duration of the principle impact; for example, $\mathrm{HIC}_{15}$ integrates the signal over a $15-\mathrm{ms}$ interval. Thus, it is essential that the main features of the impacts are also captured adequately by the iMG sensors. To that end, RMS and NRMS errors of the time-series data were calculated utilising the methodology of Camarillo et al. [6]. In that study, the RMS error was calculated over a 25-ms period, centred on the impact maximum, which was assumed to capture the relevant portion of the impact trace. In the present study, we calculated the RMS (and NRMS) error over the impact portion of the measured trace. The mean RMS (and NRMS) error for the linear acceleration and rotational velocity was $4.3 \pm 3.5 \mathrm{~g}(13.1 \pm 9.9 \%)$ and $0.7 \pm 0.4 \mathrm{rad} / \mathrm{s}$ $(3.9 \pm 2.8 \%)$, respectively. These errors are comparable with those reported by Camarillo et al. [6], where the mean RMS (and NRMS) errors for the linear acceleration and rotational velocity were $3.9 \pm 2.1 \mathrm{~g}(9.9 \pm 4.4 \%)$ and $1.0 \pm 0.8 \mathrm{rad} / \mathrm{s}$ $(10.4 \pm 9.9 \%)$, respectively, calculated over a $25-\mathrm{ms}$ interval [6]. Thus, in addition to the correlation of the peak impact values, the small RMS error between the measured timeseries data (both linear acceleration and rotational velocity) indicates high comparability of the overall shape of the waveforms measured by the two sensor systems.

The iMG sensors, sampling at $1 \mathrm{kHz}$ (linear acceleration) and $952 \mathrm{~Hz}$ (rotational velocity), measured collision metrics comparable with those recorded by an ATD, sampling at $20 \mathrm{kHz}$, whilst utilising the pendulum-based impactor. Work conducted by $\mathrm{Wu}$ et al. [64] and Nevins et al. [65] have demonstrated the importance of sampling frequency as the duration of the impact decreases. Wu et al. [64] investigated the effect of sample rate for wearable inertial sensors by dropping cadaver heads from a single height. The experiments recorded peak accelerations ranging from approximately 100-200 g for helmeted and un-helmeted situations. The authors highlighted that higher bandwidths are required for un-helmeted head impacts, recommending minimum accelerometer and gyroscope bandwidths of $500 \mathrm{~Hz}$ in unhelmeted sports conditions [64]. Nevins et al. [65] produced ATD head accelerations with magnitudes ranging from 18.4 to $194.0 \mathrm{~g}$, with the higher end achieved through impact velocities of $30 \mathrm{~m} / \mathrm{s}$ and impact durations of 1-2 ms. They demonstrated that characterising the initial acceleration and rotation peaks, at a frequency of $1 \mathrm{kHz}$, will result in undersampling of the signal, potentially underestimating the true PLA and PRV values of the ATD headform.

As highlighted, there were fundamental limitations in this study. The pendulum apparatus only provided a finite range over which controlled impacts could be assessed (10-102 g, linear acceleration). As a consequence, the impact duration was longer when compared to analogous studies that employed impactors or pneumatic cannons to mediate head acceleration and rotations [40]. Furthermore, at head accelerations greater than $70 \mathrm{~g}$, the pendulum swing was not consistently true, producing multiple collisions which resulted in three outliers. A further limitation, initially highlighted by Camarillo et al. [6], suggests that due to the highly controlled laboratory settings of such experiments, sources of potential noise mediated by humans is currently not accounted for; for example, relative mandible motion during impact. Similarly, this study assumed rigid coupling between the $\mathrm{iMG}$ and the ATD headform, a supposition that was confirmed by (1) thoroughly fastening the iMG to the ATD headform, (2) checking for relative movement after every collision and (3) analysing the measured time-series data for systematic errors such as high frequency noise (see Fig. 3). The iMGs are customised for an individual, where dental impressions are utilised to maximise coupling between the iMG and the user's maxilla. Future studies will investigate the validity of the assumption of rigid coupling between the iMG and the user's maxilla.

\section{Conclusion}

The results of this study indicate that PLA and PRV values, as well as the shape of the measured waveforms, are comparable between the two measurement systems, namely the iMG and ATD. The experimental set-up employed had 
physical constraints that only permitted collisions with relatively slow impact velocities and consequently long impact durations to be assessed. Future studies will utilise impactors or pneumatic cannons to increase the impact velocity and decrease the impact duration to address this issue. However, despite this limitation, the results recorded compared favourably with analogous experiments that studied the measurement capabilities of alternative instrumented mouthguards over similar linear acceleration and angular velocity ranges. This work presents a foundation on which to investigate HIE in professional rugby union. In addition, it delivers a means to assess coupling efficacy of the iMG attachment to an individual's jaw under a range of high-acceleration movements and collisions, which could be expanded to elucidate on position specific impact loads during training and competitive play.

Acknowledgements This research was funded by Sports and Wellbeing Analytics Limited-Grant number EGR1193. The authors would like to thank the two anonymous reviewers and the editor for their suggestions and comments, which have resulted in an improved manuscript.

Funding This research was funded by Sports and Wellbeing Analytics Limited. Sports and Wellbeing Analytics Limited have been granted patents for (1) novel electronic architecture (GB2570726), (2) system protocols (GB257076), and (3) associated firmware criteria (GB2572677). OPRO make the surrounding mouthguard while Sports and Wellbeing Analytics Limited provide parylene coated electronics.

\section{Compliance with ethical standards}

Conflict of interest The authors declare no conflict of interest.

Open Access This article is licensed under a Creative Commons Attribution 4.0 International License, which permits use, sharing, adaptation, distribution and reproduction in any medium or format, as long as you give appropriate credit to the original author(s) and the source, provide a link to the Creative Commons licence, and indicate if changes were made. The images or other third party material in this article are included in the article's Creative Commons licence, unless indicated otherwise in a credit line to the material. If material is not included in the article's Creative Commons licence and your intended use is not permitted by statutory regulation or exceeds the permitted use, you will need to obtain permission directly from the copyright holder. To view a copy of this licence, visit http://creativecommons.org/licenses/by/4.0/.

\section{References}

1. RFU (2018) England professional rugby union injury surveillance project: 2016-2017 report. In: englandrugby.com

2. Zazryn T, Cameron P, McCrory P (2006) A prospective cohort study of injury in amateur and professional boxing. Br J Sports Med 40:670-674. https://doi.org/10.1136/bjsm.2006.025924

3. O'Connor KL, Baker MM, Dalton SL et al (2017) Epidemiology of sport-related concussions in high school athletes: National Athletic Treatment, Injury and Outcomes Network (NATION),
2011-2012 through 2013-2014. J Athl Train 52:175-185. https ://doi.org/10.4085/1062-6050-52.1.15

4. Alexander DG, Shuttleworth-Edwards AB, Kidd M, Malcolm CM (2015) Mild traumatic brain injuries in early adolescent rugby players: long-term neurocognitive and academic outcomes. Brain Inj 29:1113-1125. https://doi.org/10.3109/02699052.2015.10316 99

5. Bailes JE, Petraglia AL, Omalu BI et al (2013) Role of subconcussion in repetitive mild traumatic brain injury. J Neurosurg 119:1235-1245. https://doi.org/10.3171/2013.7.JNS121822

6. Camarillo DB, Shull PB, Mattson J et al (2013) An instrumented mouthguard for measuring linear and angular head impact kinematics in American Football. Ann Biomed Eng 41:1939-1949. https://doi.org/10.1007/s10439-013-0801-y

7. McKee AC, Daneshvar DH, Alvarez VE, Stein TD (2014) The neuropathology of sport. Acta Neuropathol 127:29-51

8. Meaney DF, Morrison B, Dale Bass C (2014) The mechanics of traumatic brain injury: a review of what we know and what we need to know for reducing its societal burden. J Biomech Eng 136:021008. https://doi.org/10.1115/1.4026364

9. Patton DA, McIntosh AS, Kleiven S (2015) The biomechanical determinants of concussion: finite element simulations to investigate tissue-level predictors of injury during sporting impacts to the unprotected head. J Appl Biomech 31:264-268. https://doi. org/10.1123/jab.2014-0223

10. Rowson S, Duma SM (2013) Brain injury prediction: assessing the combined probability of concussion using linear and rotational head acceleration. Ann Biomed Eng 41:873-882. https:// doi.org/10.1007/s10439-012-0731-0

11. Bartsch A, Samorezov S, Benzel E et al (2014) Validation of an "Intelligent Mouthguard" single event head impact dosimeter. Stapp Car Crash J 58:1-27

12. Beckwith JG, Greenwald RM, Chu JJ et al (2013) Timing of concussion diagnosis is related to head impact exposure prior to injury. Med Sci Sports Exerc 45:747-754. https://doi.org/10.1249/ MSS.0b013e3182793067

13. Broglio SP, Guskiewicz KM, Norwig J (2017) If you're not measuring, you're guessing: the advent of objective concussion assessments. J Athl Train 52:160-166. https://doi. org/10.4085/1062-6050-51.9.05

14. McCrea M, Meier T, Huber D et al (2017) Role of advanced neuroimaging, fluid biomarkers and genetic testing in the assessment of sport-related concussion: a systematic review. Br J Sports Med 51:919-929

15. Wu LC, Nangia V, Bui K et al (2016) In vivo evaluation of wearable head impact sensors. Ann Biomed Eng 44:1234-1245. https ://doi.org/10.1007/s10439-015-1423-3

16. King D, Hecimovich M, Clark T, Gissane C (2017) Measurement of the head impacts in a sub-elite Australian rules football team with an instrumented patch: an exploratory analysis. Int J Sport Sci Coach 12:359-370. https://doi.org/10.1177/1747954117710512

17. King D, Hume PA, Brughelli M, Gissane C (2015) Instrumented mouthguard acceleration analyses for head impacts in amateur rugby union players over a season of matches. Am J Sports Med 43:614-624. https://doi.org/10.1177/0363546514560876

18. King DA, Hume PA, Gissane C et al (2016) The influence of head impact threshold for reporting data in contact and collision sports: systematic review and original data analysis. Sport Med 46:151-169. https://doi.org/10.1007/s40279-015-0423-7

19. Carey L, Stanwell P, Terry DP et al (2019) Verifying head impacts recorded by a wearable sensor using video footage in rugby league: a preliminary study. Sport Med Open 5:1-11. https://doi. org/10.1186/s40798-019-0182-3

20. Kimpara H, Iwamoto M (2012) Mild traumatic brain injury predictors based on angular accelerations during impacts. Ann Biomed Eng 40:114-126. https://doi.org/10.1007/s10439-011-0414-2 
21. Gadd CW (1966) Use of a weighted-impulse criterion for estimating injury hazard. In: SAE technical papers. SAE International

22. Henn H-W (1998) Crash tests and the head injury criterion. Teach Math Appl 17:162-170. https://doi.org/10.1093/teamat/17.4.162

23. Hutchinson J, Kaiser MJ, Lankarani HM (1998) The Head Injury Criterion (HIC) functional. Appl Math Comput 96:1-16. https:// doi.org/10.1016/S0096-3003(97)10106-0

24. Kleiven S, Peloso PM, von Holst H (2003) The epidemiology of head injuries in Sweden from 1987 to 2000. Inj Control Saf Promot 10:173-180. https://doi.org/10.1076/icsp.10.3.173.14552

25. Greenwald RM, Gwin JT, Chu JJ, Crisco JJ (2008) Head impact severity measures for evaluating mild trauma brain injury risk exposure. Neurosurgery 62:789-798. https://doi.org/10.1227/01. neu.0000318162.67472.ad

26. Brolinson PG, Manoogian S, McNeely D et al (2006) Analysis of linear head accelerations from collegiate football impacts. Curr Sports Med Rep 5:23-28. https://doi.org/10.1007/s1193 2-006-0025-0

27. Mihalik JP, Guskiewicz KM, Marshall SW et al (2012) Head impact biomechanics in youth hockey: comparisons across playing position, event types, and impact locations. Ann Biomed Eng 40:141-149. https://doi.org/10.1007/s10439-011-0405-3

28. Greenwald RM, Chu JJ, Crisco JJ, Finkelstein JA (2003) Head impact telemetry system (HITS) for measurement of head acceleration in the field. In: Proceedings of the American Society of biomechanics annual meeting

29. Crisco JJ, Greenwald RM, Chu JJ (2002) A novel algorithm for estimating head impact magnitude and location. In: IV World congress of biomechanics. Calgary

30. Beckwith JG, Chu JJ, Greenwald RM (2006) Development and validation of the head impact telemetry system ${ }^{\mathrm{TM}}$ for use in amateur boxing. J Biomech 39:S153. https://doi.org/10.1016/ s0021-9290(06)83517-7

31. Hanlon E, Bir C (2010) Validation of a wireless head acceleration measurement system for use in soccer play. J Appl Biomech 26:424-431. https://doi.org/10.1123/jab.26.4.424

32. Allison MA, Kang YS, Bolte JH et al (2014) Validation of a helmet-based system to measure head impact biomechanics in ice hockey. Med Sci Sport Exerc 46:115-123. https://doi. org/10.1249/MSS.0b013e3182a32d0d

33. Crisco JJ, Chu JJ, Greenwald RM (2005) An approach to calculating linear head accelerations is not affected by rotational head accelerations. In: Proceedings of the 20th congress of the international society of biomechanics

34. Manoogian S, McNeely D, Duma S, et al (2006) Head acceleration is less than $10 \%$ of helmet acceleration in football impacts. In: Biomedical sciences instrumentation. pp 383-388

35. Beckwith JG, Greenwald RM, Chu JJ (2012) Measuring head kinematics in football: correlation between the head impact telemetry system and hybrid III headform. Ann Biomed Eng 40:237-248. https://doi.org/10.1007/s10439-011-0422-2

36. GForceTracker (2016) How it works. GForceTracker, Richmond Hill

37. Guskiewicz KM, Mihalik JP (2011) Biomechanics of sport concussion. Exerc Sport Sci Rev 39:4-11. https://doi.org/10.1097/ JES.0b013e318201f53e

38. Murayama H, Hitosugi M, Motozawa Y et al (2020) Biomechanical analysis of the head movements of a person thrown by the judo technique 'Seoi-nage'. Neurol Med Chir (Tokyo) 60:101-106. https://doi.org/10.2176/nmc.oa.2019-0206

39. King D, Hume P, Gissane C, Clark T (2017) Head impacts in a junior rugby league team measured with a wireless head impact sensor: an exploratory analysis. J Neurosurg Pediatr 19:13-23. https://doi.org/10.3171/2016.7.PEDS1684

40. Nevins D, Smith L, Kensrud J (2015) Laboratory evaluation of wireless head impact sensor. Proced Eng 112:175-179
41. Paris AJ, Antonini KR, Brock JMF (2010) Accelerations of the head during soccer ball heading. In: ASME 2010 summer bioengineering conference, SBC 2010. American Society of Mechanical Engineers Digital Collection, pp 815-816

42. Kara TM, DelSignore JA, Brock JM, et al (2012) Evaluation of an instrumented mouthguard to measure the accelerations of the head due to soccer ball heading. In: Proceedings of the 12th Pan-American congress of applied mechanics. Port of Spain; Trinidad

43. Bartsch A, Samorezov S (2013) Cleveland clinic intelligent mouthguard: a new technology to accurately measure head impact in athletes and soldiers. In: Southern ŚO (ed) Sensing technologies for global health, military medicine, and environmental monitoring III. SPIE, Bellingham, p 87230N

44. Hernandez F, Shull PB, Camarillo DB (2015) Evaluation of a laboratory model of human head impact biomechanics. J Biomech 48:3469-3477. https://doi.org/10.1016/j.jbiom ech.2015.05.034

45. Hernandez F, Wu LC, Yip MC et al (2015) Six degree-offreedom measurements of human mild traumatic brain injury. Ann Biomed Eng 43:1918-1934. https://doi.org/10.1007/s1043 9-014-1212-4

46. Kleiven S (2002) Finite element modelling of the human head. Royal Institute of Technology, Stockholm

47. Siegmund GP, Guskiewicz KM, Marshall SW et al (2016) Laboratory validation of two wearable sensor systems for measuring head impact severity in football players. Ann Biomed Eng 44:1257-1274. https://doi.org/10.1007/s10439-015-1420-6

48. Siegmund GP, Guskiewicz KM, Marshall SW et al (2014) A headform for testing helmet and mouthguard sensors that measure head impact severity in football players. Ann Biomed Eng 42:1834-1845. https://doi.org/10.1007/s10439-014-1052-2

49. BBC (2019) RFU tackle height trial ended after concussions rise in Championship Cup. In: BBC. https://www.bbc.co.uk/sport/ rugby-union/47000468

50. World Rugby (2019) World Rugby Laws. https://laws.world rugby.org/

51. Bartsch A, Benzel E, Miele V et al (2012) Hybrid III anthropomorphic test device (ATD) response to head impacts and potential implications for athletic headgear testing. Accid Anal Prev 48:285-291. https://doi.org/10.1016/j.aap.2012.01.032

52. Backaitis SH, Mertz HJ (1993) Hybrid III: The First Human-like Crash Test Dummy. Society of Automotive Engineers, Warrendale, PA, p 830

53. Chrisman SPD, Mac Donald CL, Friedman S et al (2016) Head impact exposure during a weekend youth soccer tournament. J Child Neurol 31:971-978. https://doi.org/10.1177/0883073816 634857

54. King DA, Hume PA, Gissane C et al (2018) Head impact exposure from match participation in women's rugby league over one season of domestic competition. J Sci Med Sport 21:139-146. https://doi.org/10.1016/j.jsams.2017.10.026

55. King DA, Hume PA, Gissane C, Clark TN (2017) Semi-professional rugby league players have higher concussion risk than professional or amateur participants: a pooled analysis. Sport Med 47:197-205. https://doi.org/10.1007/s40279-016-0576-z

56. Oliphant TE (2007) Python for scientific computing. Comput Sci Eng 9:10-20. https://doi.org/10.1109/MCSE.2007.58

57. Rowson S, Beckwith JG, Chu JJ et al (2011) A six degree of freedom head acceleration measurement device for use in football. J Appl Biomech 27:8-14

58. Atkinson G, Nevill AM (1998) Statistical methods for assessing measurement error (reliability) in variables relevant to sports medicine. Sport Med 26:217-238. https://doi. org/10.2165/00007256-199826040-00002 
59. Atkinson G, Nevill AM, Edwards B (1999) What is an acceptable amount of measurement error? The application of meaningful 'analytical goals' to the reliability of sports science measurements made on a ratio scale. J Sports Sci 17:18

60. Baumgartner TA, Chung H (2001) Confidence limits for intraclass reliability coefficients. Meas Phys Educ Exerc Sci 5:179188. https://doi.org/10.1207/S15327841MPEE0503_4

61. Bland MJ, Altman DG (1986) Statistical methods for assessing agreement between two methods of clinical measurement. Lancet 327:307-310. https://doi.org/10.1016/S0140-6736(86)90837 $-8$

62. Stephens VM, Bacon DGC (1993) The development of a method for dynamic simulation of side impacts using a hyge accelerator-the s.I.D.E. procedure. In: Proceedings: International technical conference on the enhanced safety of vehicles. National Highway Traffic Safety Administration, pp 668-673

63. Hopton JR, Payne AR (1996) Comparison study of EuroSID, USSID, BioSID performance using MIRA's new M-SIS side impact simulation technique. In: SAE technical papers. SAE International
64. Wu LC, Laksari K, Kuo C et al (2016) Bandwidth and sample rate requirements for wearable head impact sensors. J Biomech 49:2918-2924. https://doi.org/10.1016/j.jbiomech.2016.07.004

65. Nevins D, Hildenbrand K, Kensrud J et al (2018) Laboratory and field evaluation of a small form factor head impact sensor in un-helmeted play. Proc Inst Mech Eng Part P J Sport Eng Technol 232:242-254. https://doi.org/10.1177/1754337117739458

66. Mital NK, King AI (1979) Computation of rigid-body rotation in three-dimensional space from body-fixed linear acceleration measurements. J Appl Mech Trans ASME 46:925-930. https:// doi.org/10.1115/1.3424679

Publisher's Note Springer Nature remains neutral with regard to jurisdictional claims in published maps and institutional affiliations. 